\section{Streptococcus dysgalactiae endocarditis presenting as acute endophthalmitis}

\author{
Angelina Su-Min Yong, Su Yin Lau, \\ Tsung Han Woo, Jordan Yuanzhi Li, \\ Tuck Yean Yong \\ Department of General Medicine, \\ Flinders Medical Centre and Faculty \\ of Health Science, Flinders University, \\ Bedford Park, South Australia.
}

\begin{abstract}
Endogenous endophthalmitis is a rare ocular infection affecting the vitreous and/or aqueous humours. It is associated with poor visual prognosis and its commonest endogenous aetiology is infective endocarditis. The causative organisms of endogenous endophthalmitis complicating endocarditis are mainly Group A or B streptococci. The identification of Group $\mathrm{C}$ and G streptococci such as Streptococcus dysgalactiae is comparatively uncommon and has only been reported in a few case reports or series. We therefore report a case of infective endocarditis caused by Streptococcus dysgalactiae first presenting with endogenous endophthalmitis, the most likely source being osteomyelitis of both feet in a patient with type I diabetes. The patient was treated with a course of intravenous benzylpenicillin, intravitreal antibiotics, bilateral below knee amputations and mitral valve replacement. She survived all surgical procedures and regained partial visual acuity in the affected eye.
\end{abstract}

\section{Introduction}

Endophthalmitis refers to bacterial or fungal infection within the eye involving the vitreous and/or aqueous humours. Acute bacterial endophthalmitis is a vision-threatening condition requiring emergency management. Most cases of endophthalmitis are exogenous in aetiology from external inoculation of organisms such as trauma, eye surgery and extension of corneal injury. However, endogenous bacterial endophthalmitis can occur infrequently resulting from seeding during bacteraemia. In one case series, endocarditis was the most frequent cause of endogenous endophthalmitis, accounting for $40 \%$ of cases. ${ }^{1}$ Among $\beta$-haemolytic streptococci, the more common causative organisms have been Groups A or B streptococci. ${ }^{2}$ Other streptococcal organisms such as those from groups $\mathrm{C}$ and $\mathrm{G}$ have been rarely reported in endogenous endophthalmi- tis complicating endocarditis. ${ }^{3}$

We report a case of endogenous endophthalmitis in the setting of mitral valve endocarditis and non-healing diabetic foot ulcers caused by Streptococcus dysgalactiae ( $S$. dysgalactiae). The literature on this subject is also reviewed.

\section{Case Report}

A 70-year-old woman presented with a five day history of painful left eye, deterioration in visual acuity and fever $\left(37.8^{\circ} \mathrm{C}\right)$. She did not have any preceding history of trauma or surgery to her eyes. Her medical history included type I diabetes (glycohaemoglobin prior to admission was $7.5 \%$ ) complicated by proliferative retinopathy; Charcot's neuroarthropathy in both feet; non-healing ulcers on the plantar surface of her feet for at least a year prior to this presentation; surgically treated Graves' disease; hypertension and hyperlipidaemia. She also had a history of anaphylactic reaction to penicillin.

She was apyrexial on arrival at our institution, having received three days of oral cephalexin prescribed by her family doctor. Her blood pressure was 135/85 mmHg; pulse rate was $72 \mathrm{bpm}$ with a regular rhythm and oxygen saturation $95 \%$ on room air. She had marked bilateral conjunctival injection and loss of peripheral vision. Her visual acuity was $6 / 38$ in the right eye and 6/120 in the left. An hypopyon was present in the left eye and severe diabetic proliferative retinopathy was evident in both eyes. She did not have splinter haemorrhages or any other peripheral stigmata of infective endocarditis. On cardiac auscultation, there was a pansystolic murmur, consistent with mitral regurgitation. Urinalysis showed blood (trace amounts) and protein (30 $\mathrm{mg} / \mathrm{dL}$ ). Her haematological and biochemical parameters are shown in Table 1.

Ophthalmology review confirmed the diagnosis of endophthalmitis. Bilateral vitreous aspiration was performed and intravitreal antibiotics (vancomycin and ceftazidime) were administered. A set of blood culture taken before administration of oral antibiotics revealed growth of Group $\mathrm{G}$ streptococcus, $S$ dysagalactiae. The organism was susceptible to penicillin with a minimum inhibitory concentration (MIC) of $0.06 \mu \mathrm{g} / \mathrm{mL}$. Cultures from the intravitreal aspirate were negative. Chest Xray revealed a small left pleural effusion with associated left lung basal collapse. Magnetic resonance imaging (MRI) of both feet revealed evidence of chronic osteomyelitis (Figure 1). Transthoracic echocardiography showed severe mitral regurgitation and a large mobile vegetation measuring $2 \mathrm{~cm}$ in length and 0.9 $\mathrm{cm}$ in diameter. This was associated with mod-
Correspondence: Tuck Y. Yong, Department of General Medicine, Flinders Medical Centre, Flinders Drive, Bedford Park, South Australia 5042.

Tel. +61.8.8204.6249 - Fax: +61.8.8204.6979.

E-mail: tuck.yong@health.sa.gov.au

Key words: Endocarditis, endophthalmitis, Group C streptococcus, Group G streptococcus, streptococcus dysgalactiae.

Acknowledgement: we acknowledge the multidisciplinary contributions from anaesthetic, cardiology, cardiothoracic surgery, endocrinology, general medicine, immunology, infectious diseases, intensive care, occupational therapy, ophthalmology, physiotherapy, radiology, rehabilitation and vascular surgery departments in managing this patient during her complex admission.

Conflict of interest: the authors report no conflicts of interest.

Received for publication: 4 October 2011. Revision received: 30 December 2011.

Accepted for publication: 8 January 2012.

This work is licensed under a Creative Commons Attribution NonCommercial 3.0 License (CC BYNC 3.0).

(C) Copyright A. Su-Min Yong et al., 2012

Licensee PAGEPress srl, Italy

Infectious Disease Reports 2012; 4:e16

doi:10.4081/idr.2012.e16

erate tricuspid regurgitation and an estimated pulmonary artery pressure of $85 \mathrm{mmHg}$. The transoesophageal echocardiography confirmed two large vegetations $(1.6 \times 1.1 \mathrm{~cm}$ and another elongated mass of $1.5 \mathrm{~cm}$ ) on the posterior leaflet of the mitral valve with associated complex severe mitral regurgitation (Figure 2). No vegetations were seen on the other heart valves. She also underwent a coronary angiography, which revealed a $70 \%$ proximal left anterior descending artery stenosis and $80 \%$ stenosis to the diagonal vessel.

The patient was commenced on an 8-week course of intravenous antibiotics, initially with intravenous cephazolin $2 \mathrm{~g}$ four times a day. This was later changed to intravenous benzylpenicillin $1.8 \mathrm{~g}$ four times a day after receiving penicillin desensitization under the supervision of the immunology team. In addition to intravitreal antibiotics, her left eye was treated with topical gentamicin (1.4\%) and cephazolin (1\%), one drop each, four times a day and atropine (1)\% 1 drop twice a day. With the treatment regimen listed above, her visual acuity improved to $6 / 36$ in the affected left eye. Her cardiac function was optimised by increasing the dose of trandolapril and introduction of frusemide $40 \mathrm{mg}$ daily.

During week 4 of her hospital admission, 
she underwent bilateral below knee amputations to eradicate the potential source of infection prior to cardiac surgery. Culture from the surgical bone specimens of osteomyelitis did not isolate any organisms. At week 7 , she underwent concomitant porcine tissue mitral valve replacement and engraftment of left internal mammary artery to the left anterior descending coronary artery. She recovered from both surgeries without any major complications. At week 9, she was transferred to a rehabilitation facility for ongoing recovery and transition to going home. At two months after discharge, she remained clinically well.

\section{Discussion}

Infective endocarditis is a life-threatening condition and in-hospital mortality can be as high as 20\%. ${ }^{4}$ Groups A and B streptococci are common causative organism in infective endocarditis while groups $\mathrm{C}$ and $\mathrm{G}$ account for less than $2 \%$ of cases. $^{3} S$. dysgalactiae is a Lancefield group $\mathrm{C}$ or $\mathrm{G}$ streptococcus. This bacterium primarily causes skin and soft-tissue infections including pyoderma, cellulitis, wound infections, abscesses, erysipelas and necrotizing fasciitis. ${ }^{3}$ However invasive infections can also occur resulting in septic arthritis, osteomyelitis, pleuropulmonary infections, meningitis, streptococcal toxic-like syndrome and endocarditis.

Endocarditis caused by group $\mathrm{G}$ streptococcus has been reported few times in the literature. ${ }^{5-7} S$. dysgalactiae can be divided into five distinct subtypes. ${ }^{8}$ Human disease is most frequently associated with $S$. dysgalactiae subspecies equisimilis, Lancefield serogroup G. ${ }^{8}$ Concomitant occurrence of endocarditis and endophthalmitis caused by $S$. dysgalactiae has previously been reported in two other cases. ${ }^{9,10}$ In both cases the vegetations were present on the mitral valve; one case had bilateral endophthalmitis while the other was unilateral. Another report had a case of group $G$ streptococcal endocarditis complicated by bilateral endophthalmitis but the specific species was not reported. ${ }^{11}$

Endogenous bacterial endophthalmitis accounts $2-15 \%$ of all endophthalmitis cases. ${ }^{12}$ The causative organism of endogenous endophthalmitis varies with geographical regions. In North America and Europe, streptococci, Staphylococcus aureus and gram-negative bacilli are the common organisms identified, while in Asia, gram-negative bacilli, especially Klebsiella and Escherichia coli, predominate. ${ }^{13,14}$ The present case demonstrates the importance of recognizing extra-cardiac manifestations of infective endocarditis which generally result from thromboembolism. Other well-known ocular manifestations of endo-
Table 1. Haematological and biochemical parameters.

\begin{tabular}{lcc} 
& Value on presentation & Reference range \\
Haemoglobin, $g / \mathrm{L}$ & 109 & $115-155$ \\
White cell count, $\times 10^{9} / \mathrm{L}$ & 13.5 & $4.00-11.0$ \\
\hline Platelet count, $\times 10^{6} / \mathrm{L}$ & 244 & $150-400$ \\
Neutrophil count, $\times 10^{9} / \mathrm{L}$ & 14.4 & $1.80-7.50$ \\
\hline Serum sodium, $\mu \mathrm{mol} / \mathrm{L}$ & 138 & $137-145$ \\
Serum potassium, $\mu \mathrm{mol} / \mathrm{L}$ & 4.1 & $3.2-4.3$ \\
\hline Serum urea, $\mu \mathrm{mol} / \mathrm{L}$ & 3.6 & $2.7-8.0$ \\
Serum creatinine, $\mu \mathrm{mol}$ & 61 & $50-100$ \\
\hline Lactate dehydrogenase, $\mathrm{U} / \mathrm{L}$ & 335 & $110-230$ \\
C-reactive protein, $\mathrm{g} / \mathrm{L}$ & 75 & $<10$
\end{tabular}



Figure 1. Magnetic resonance imaging of both feet showing advanced Charcot joint with complete distortion of the normal anatomy. (A) On the left, an ulcer (arrow) is present on the plantar aspect of the mid/hindfoot with enhancing tissue extending to the bases of the lateral midfoot joints and previous intramedullary rod defect in the distal tibial metaphysis, suggestive of infection. (B) On the right, a large plantar ulcer (arrow) and associated osteomyelitis in the calcaneum, head of talus, remaining cuneiforms, and lateral metatarsal bases.

carditis include Roth's spots which are haemorrhagic spots in the conjunctiva, and retinal haemorrhage.

From the limited data available, $S$. dysgalactiae seems to infect left-sided native heart valves after gaining entry through the skin and is associated with a high prevalence of complications. In the current case, the primary source of infection was most likely the nonhealing ulcers on the feet and the associated chronic osteomyelitis leading to more invasive infection. The current literature suggests an association between group $\mathrm{G}$ streptococcal bacteraemia or other invasive infections and underlying immunosuppressive conditions such as diabetes mellitus, chronic alcoholism, cardiovascular disease and malignancies. ${ }^{15}$

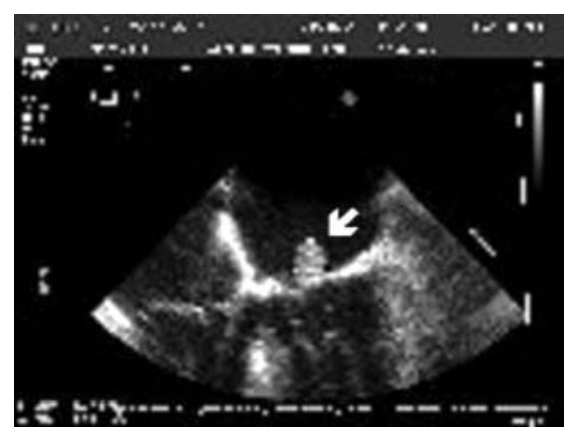

Figure 2. The transoesophageal echocardiography showing one of the two large vegetations (one was $1.6 \times 1.1 \mathrm{~cm}$, arrow) on the posterior leaflet of mitral valve with associated complex severe mitral regurgitation. 
Endogenous endophthalmitis requires emergency treatment because a delay can result in permanent visual loss in the affected eye.

Reliable identification of $S$. dysgalactiae has been enhanced by the use of sequence analysis of the 16S rRNA gene and phylogenetic analysis shows that it is most closely related to this organism within the pyogenic group of streptococci. ${ }^{16}$ This was used to identify S. dysgalacti$a e$ in vitreous aspirate from the two aforementioned cases. ${ }^{9} 10$ For subspecies information on $S$. dysgalactiae, the analysis of the 23 rRNA appeared to better distinguish subspecies $d y s$ galactiae from equisimilis. ${ }^{17}$

With regards to treatment, $\mathrm{S}$. dysgalactiae isolates remain almost uniformly susceptible to penicillin and other $\beta$-lactam agents. Therefore, penicillin is considered the drug of choice. Isolates with slightly increased MIC of $0.25 \mu \mathrm{g} / \mathrm{mL}$ for penicillin were recently reported. ${ }^{18}$ The clinical significance of this intermediate susceptibility remains uncertain. ${ }^{19}$ In current case, due to a previous penicillin hypersensitivity reaction, the patient underwent penicillin desensitisation thus allowing intravenous benzylpenicillin to be utilised with great success. The present case also demonstrates that in addition to antibiotic therapy, it is important to eradicate any source of ongoing infection prior to valve replacement surgery. Bilateral below-knee amputations were required in our case due to concerns of chronic osteomyelitis in her feet.

The prognosis of $S$. dysgalactiae endocarditis complicated by endogenous endophthalmitis remains unknown. In the two cases reported, one patient had a poor visual outcome while the endocarditis was successfully treated and the other patient's outcome after treatment was not reported.

In summary, this case demonstrates the importance of recognising extra-cardiac manifestations of infective endocarditis, such as endophthalmitis and the identification of an uncommon causative organism, $S$. dysgalacti$a e$, in this setting. Endogenous endophthalmitis complicating infective endocarditis requires urgent and intensive treatment to prevent visual loss and other cardiac or systemic complications.

\section{References}

1. Okada AA, Johnson RP, Liles WC, et al. Endogenous bacterial endophthalmitis. Report of a ten-year retrospective study. Opthalmology 1994;101:832-8.

2. Baddour LM. Infective endocarditis caused by beta-hemolytic streptococci. The Infectious Diseases Society of America's Emerging Infections Network. Clin Infect Dis 1998;26:66-71.

3. Broyles LN, Van Beneden C, Beall B, et al. Population-based study of invasive disease due to $\beta$-hemolytic streptococci of groups other than A and B. Clin Infect Dis 2009;48: 706-12.

4. Wallace SM, Walton BI, Kharbanda RK, et al. Mortality from infective endocarditis: clinical predictors of outcome. Heart 2002; 88:53-60.

5. Bouza E, Meyer RD, Busch DF. Group G streptococcal endocarditis. Am J Clin Path 1978;70:108-11.

6. Smyth EG, Pallett AP, Davidson RN. Group G streptococcal endocarditis: two case reports, review of the literature and recommendations for treatment. J Infect 1988;16: 169-76.

7. Bucher A, Gaustad P. Septicaemia and endocarditis caused by group $\mathrm{G}$ streptococci in a Norwegian hospital. Eur J Clin Microbiol Infect Dis 1990;9:251-6.

8. Vandamme P, Pot B, Falsen E, et al. Taxonomic study of Lancefield streptococcal group C, G, and L (Streptococcus dysgalactiae) and proposal of $\mathrm{S}$. dysgalactiae subsp. equisimilis subsp. nov. Int $\mathrm{J}$ Syst Bacteriol 1996;46:774-81.

9. Wickramasinghe N, Harris K. Bilateral endophthalmitis as a primary manifestation of Streptococcus dysgalactiae endocarditis and the role of $16 \mathrm{~S}$ rDNA polymerase chain reaction in identification. Diagn Microbiol Infect Dis 2010;67:185-7.

10. Suemori S, Sawada A, Komori S, et al. Case of endogenous endophthalmitis caused by Streptococcus equisimilis. Clin Ophthalmol 2010;4:917-8.

11. Gopalamurugan $A B$, Wheatcroft $S$, Hunter
P, Thomas MR. Bilateral endophthalmitis and ARDS complicating group G streptococcal endocarditis. Lancet 2005;366:2062.

12. Shrader SK, Band JD, Lauter CB, Murphy P. The clinical spectrum of endophthalmitis: incidence, predisposing factors, and features influencing outcomes. J Infect Dis 1990;162:115-20.

13. Wong JS, Chan TK, Lee HM, Chee SP. Endogenous bacterial endophthalmitis: an east Asian experience and a reappraisal of a severe ocular affliction. Ophthalmology 2000;107:1483-91.

14. Jackson TL, Eykyn SJ, Graham EM, Stanford MR. Endogenous bacterial endophthalmitis: a 17-year prospective series and review of 267 reported cases. Surv Ophthalmol 2003;48:403-23.

15. Armstrong D, Blevins A, Louria DB, et al. Groups B, C and G streptococcal infections in a cancer hospital. Ann N Y Acad Sci 1970;174:511-22.

16. Facklam R. What happened to the streptococcal: Overview of taxonomic and nomenclature changes. Clin Microbiol Rev 2002; 15:613-30.

17. Kawata K, Minakami T, Mori Y, et al. rDNA sequence analyses of Steptococcus dysgalactiae subsp. equisimilis isolates from pigs. Int J Syst Evol Microbiol 2003;53: 1941-6.

18. Biedenbach DJ, Toleman MA, Walsh TR, Jones RN. Characterization of fluoroquinolone-resistant $\beta$-hemolytic Streptococcus spp. Isolated in North America and Europe including the first report of fluoroquinolone-resistant Streptococcus dysgalactiae subspecies equisimilis: report from the SENTRY Antimicrobial Sur-veillance Program (1997-2004). Diagn Microbiol Infect Dis 2006;55:119-27.

19. Brandt CM, Spellerberg B. Human infections due to Streptococcus dysgalactiae subspecies equisimilis. Clin Infect Dis 2009;49:766-72. 